\title{
Bevacizumab: a new way of doing business. Part 2
}

\author{
Andrew J. Lotery $\mathbb{D}^{1} \cdot$ Michael A. Burdon ${ }^{2}$
}

Received: 30 October 2018 / Accepted: 7 November 2018 / Published online: 27 November 2018

(c) The Royal College of Ophthalmologists 2018

In 2006, I (AJL) co-wrote an editorial in Eye entitled 'Bevacizumab: a new way of doing business' [1]. This article described how an unlicensed drug, bevacizumab, had recently been reported to be very effective in treating "wet AMD". This editorial pointed out that bevacizumab uptake was rapid due to its low price, but that clinical trials were needed to ensure it was safe. Since then two landmark clinical trials, IVAN performed in the UK and CATT performed in the USA, confirmed the efficacy of bevacizumab when compared to ranibizumab [2]. These trials were challenging to do and to achieve with public money. Despite these data, due to various regulatory hurdles bevacizumab was not adopted in the NHS. These challenges were discussed in an editorial I (AJL) wrote with our then college President Prof MacEwen [3]. This editorial was cited by NHS commissioners when they wrote to the Secretary of State for Health asking to be allowed to use bevacizumab due to cost press. The response from the Department of Health was that "It would not only be unlawful but against the wider public interest if ministers were to attempt to set aside that licensing system in order purely to cut costs." [4]. This would seem to be the end of the matter, but the increasing challenge of delivering health care equitably continued to vex health commissioners when a very cheap drug, bevacizumab, was clinically as effective as ranibizumab [2].

To estimate what savings could be achieved, a group of junior ophthalmologists used freedom of information requests to identify how much bevacizumab, ranibizumab

Andrew J. Lotery

a.j.lotery@soton.ac.uk

1 Clinical and Experimental Sciences, Faculty of Medicine, University of Southampton, South Lab and Path Block, Mailpoint 806, Level D, University Hospital Southampton, Southampton SO16 6YD, Southampton, UK

2 Queen Elizabeth Hospital Birmingham, University Hospitals Birmingham, NHS Foundation Trust, Mindelsohn Way Edgbaston, Birmingham B15 2TH, UK and aflibercept were being used in the NHS in January 2015 [4]. They calculated that if all injections used divided bevacizumab this would save the NHS £449 196354 per year.

If this money was reinvested in ophthalmology, then many of our capacity problems could be addressed. A survey by the Royal College of Ophthalmologists identified that patients go blind because of delays in accessing the hospital eye service [5]. Frankly, this should be a public outcry.

Therefore, should doctors worry about the cost of the medicines they prescribe? The General Medical Council thinks yes. Their Chief Executive stated "We expect doctors to make good use of the resources available to them and sympathise with the concerns of ophthalmologists making decisions between using a cheaper product outside the terms of its license or a more expensive licensed alternative. We cannot of course give specific clinical or legal advice. But we can say that where doctors are working in partnership with patients, following clinical guidance and making prescribing decisions in good faith on the basis of evidence and experience, the use of Avastin would not cause us any concerns." [6].

There are interesting paradoxes regarding bevacizumab. In the USA it is the commonest anti-VEGF agent prescribed overall. The 2018 preferences and trends survey presented at the American Academy of Ophthalmology annual meeting noted bevacizumab was the first-line anti-VEGF agent for wet AMD for $70.2 \%$ of US ophthalmologists. In the UK it is frequently used in private health care as private health insurers will not pay for the licensed medications. The World Health Organisation lists it as an essential medicine for the treatment of AMD [7].

Therefore, it is clear there is an established market for bevacizumab, which again raises the question why can the NHS not use it? Further, NICE recently stated there are no clinically significant differences in the effectiveness and safety of anti-VEGF medications that are licensed for treating AMD and those that are not licensed, such as bevacizumab [8]. 
Therefore, a group of NHS commissioners recently asked clinicians to use bevacizumab as a treatment for AMD. They felt this was justifiable as the more expensive drugs ranibizumab and aflibercept had no increased benefit in terms of visual outcome. Two drug companies, Bayer PLC and Novartis Pharmaceuticals UK Limited, asked for a judicial review of this practice and their legal challenge was dismissed [9]. Mrs Justice Whipple, who heard the case, said that the companies' arguments that the NHS could not consider bevacizumab unless Roche applied for a licence for it as an eye treatment was an "absurd proposition." She said, "It would give unbounded power to the pharmaceutical companies to decide which medicines to make available for which purposes." She added, "That would be seriously detrimental to the wider public interest in maintaining a cost effective public health system." [10].

Clinicians are concerned with this ruling for various reasons. Some of the issues are discussed in the accompanying paper in this week's edition by Roos et al. [11]. Other issues include a concern that drug companies will no longer innovate in the field of "wet" AMD and develop novel drugs. However, there is no sign of this in the USA, where the majority of "wet" AMD patients receive bevacizumab. Another issue raised is the lack of post-marketing pharmacovigilance for an unlicensed medicine. However, bevacizumab remains subject to pharmacovigilance for its licensed use in cancer therapy.

Perhaps the greatest concern is that compared to the licensed medicines, bevacizumab will require more follow-up visits and injections. The LUCAS study showed that using a treat-and-extend approach patients required on average 8.9 injections of bevacizumab compared to eight injections of ranibizumab in a year [12]. For centres that follow the VIEW 2 protocol only seven injections of aflibercept are required [13]. So it is likely that more capacity will be required in terms of manpower and space to deliver a bevacizumab antiVEGF service. Patients will also have to be willing to have more clinic visits. However, if these issues are addressed by investment in the hospital eye service it should be possible to deliver a bevacizumab service and improve capacity in the hospital eye service. This would be a very welcome development. The Royal College of Ophthalmologists supports doctors being allowed to use bevacizumab as a choice for AMD, but only in the context that extra resources are provided to allow such a service to develop [14]. Therefore, after
12 years of debate, the recent judicial review outcome suggests that for NHS ophthalmologists bevacizumab finally represents a new way of doing business.

\section{Compliance with ethical standards}

Conflict of interest The authors declare that they have no conflict of interest.

\section{References}

1. Canning C, Lotery A. Bevacizumab: a new way of doing business? Eye. 2007;21(6):891-91.

2. Chakravarthy U, Harding SP, Rogers CA, Downes SM, Lotery AJ, Culliford LA, et al. Alternative treatments to inhibit VEGF in age-related choroidal neovascularisation: 2-year findings of the IVAN randomised controlled trial. Lancet. 2013;382(9900): 1258-67.

3. Lotery A, MacEwen C. What is stopping the NHS from using bevacizumab for macular degeneration and other retinal disorders? BMJ. 2014;349:g6887.

4. Shalaby AK, Lewis K, Bush K, Meredith PR, Di Simplicio S, Lockwood AJ. Licence to save: a UK survey of anti-VEGF use for the eye in 2015. Eye (Lond). 2016;30(11):1404-6.

5. Foot B, MacEwen C. Surveillance of sight loss due to delay in ophthalmic treatment or review: frequency, cause and outcome. Eye (Lond). 2017;31(5):771-5.

6. GMC guidance. https://www.gmc-uk.org/news/media-centre/ media-centre-archive/gmc-responds-to-new-nice-guidance.

7. WHO model list of essential medicines. http://www.who.int/ medicines/publications/essentialmedicines/en/.

8. Nice guidance. https://www.nice.org.uk/guidance/NG82.

9. Judicial review summary. https://www.judiciary.uk/wp-content/ uploads/2018/09/bayer-and-novartis-v-nhs-darlington-ccg-summa ry.pdf.

10. BMJ report of judicial review. https://www.bmj.com/content/362/ bmj.k4035.

11. Hyry HI, Roos JCP. Implications for pharmaceutical companies and clinicians from the Bayer v NHS judgement: an increasing budgetary focus for both. Eye (Lond). 2018; https://doi.org/10. 1038/s41433-018-0280-3.

12. Berg K, Pedersen TR, Sandvik L, Bragadottir R. Comparison of ranibizumab and bevacizumab for neovascular age-related macular degeneration according to LUCAS treat-and-extend protocol. Ophthalmology. 2015;122(1):146-52.

13. Almuhtaseb H, Talks JS, Johnston R, Lotery AJ. Second-year visual acuity outcomes of nAMD patients treated with aflibercept: data analysis from the UK Aflibercept Users Group. Eye (Lond). 2017;31(11):1582-8.

14. RCOpth response to avastin. https://www.rcophth.ac.uk/2018/09/ the-royal-college-of-ophthalmologists-is-delighted-that-the-highcourt-has-found-in-favour-of-the-use-of-avastin-for-wet-amd/. 